\title{
Modeling water table fluctuations by means of a stochastic differential equation
}

\author{
Marc F. P. Bierkens \\ DLO, Winand Staring Centre for Integrated Land, Soil and Water Research, Wageningen, Netherlands
}

\begin{abstract}
The combined system of soil-water and shallow groundwater is modeled with simple mass balance equations assuming equilibrium soil moisture conditions. This results in an ordinary but nonlinear differential equation of water table depth at a single location. If errors in model inputs, errors due to model assumptions and parameter uncertainty are lumped and modeled as a wide band noise process, a stochastic differential equation (SDE) results. A solution for the stationary probability density function is given through use of the Fokker-Planck equation. For the nonstationary case, where the model inputs are given as daily time series, sample functions of water table depth, soil saturation, and drainage discharge can be simulated by numerically solving the SDE. These sample functions can be used for designing drainage systems and to perform risk analyses. The parameters and noise statistics of the SDE are calibrated on time series of water table depths by embedding the SDE in a Kalman filter algorithm and using the filter innovations in a filter-type maximum likelihood criterion. The stochastic model is calibrated and validated at two locations: a peat soil with a very shallow water table and a loamy sand soil with a moderately shallow water table. It is shown in both cases that sample functions simulated with the SDE are able to reproduce a wide range of statistics of water table depth. Despite its unrealistic assumption of constant inputs, the stationary solution derived from the Fokker-Planck equation gives good results for the peat soil, most likely because the characteristic response time of the water table is very small.
\end{abstract}

\section{Introduction}

In lowland areas, such as the Netherlands, the phreatic surface is found at shallow depths, usually within $2 \mathrm{~m}$ below ground surface. Because such shallow water tables can have large impacts on crop growth and vegetation development, knowledge of the temporal and spatial variation of the water table depth (i.e., phreatic surface level relative to ground surface) is extremely important for the management of agricultural areas and nature reserves.

One way of describing the spatiotemporal variation of the phreatic surface is to use a distributed transient groundwater model. However, the spatial variation of a shallow water table is mostly determined by the properties of the unsaturated zone, and in case of the Netherlands, by a dense network of drainage ditches with controlled surface water levels. To describe such shallow groundwater tables with sufficient accuracy (standard deviation of prediction error $<0.2 \mathrm{~m}$ ), the distributed groundwater model must be of high resolution, including the unsaturated zone and all surface waters. This would require an enormous amount of input (e.g., surface elevations, spatially varying saturated and unsaturated conductivity, geometry of the surface waters) that can only be acquired at high costs.

Alternatively, one can use simple one-dimensional models of soil-groundwater interaction that describe the temporal variation of the water table at a single location. Such onedimensional models only require some meteorological inputs and simple borehole descriptions of the topsoil (of which there are many in the Netherlands). If sufficient borehole descrip-

Copyright 1998 by the American Geophysical Union.

Paper number 98WR02298 0043-1397/98/98WR-02298\$09.00 tions are available or when the spatial variation of soil profiles can be derived from a soil classification map, the onedimensional models can be used to generate long time series of daily water table depths at a large number of locations. A spatiotemporal description then follows from interpolating between these locations.

One-dimensional models that describe water table fluctuations include empirical models such as transfer function-noise models [Box and Jenkins, 1976; Hipel and McLeod, 1994; Tankersley et al., 1993; Gehrels et al., 1994; van Geer and Zuur, 1997; Knotters and Van Walsum, 1997] and physical-mechanistic models that solve Richards' equation in heterogeneous soils [Neuman et al., 1974; Belmans et al., 1983; Feddes et al., 1988]. A disadvantage of the physical-mechanistic models for unsaturated flow is that it would require a lot of effort to apply them at many locations. One reason for this difficulty is that in practical applications the data are lacking to set the many parameters of these models (e.g., unsaturated hydraulic conductivity; leaf area index and root distribution as a function of time; fraction of immobile water). A second reason is that due to numerical difficulties in solving Richards' equation, it often happens that at a location no reasonable solution is obtained. The disadvantage of empirical models such as transfer function-noise models is that they are not suitable for making predictions when the dynamical behavior of the hydrological system changes, for instance, when the drainage system is altered or there are changes in land use.

The purpose of this paper is to develop a stochastic model that captures the dynamics of shallow groundwater table fluctuations at a single location without the need to describe flow in the unsaturated zone. Although its structure is simple, the model can be used to make predictions when local changes of 


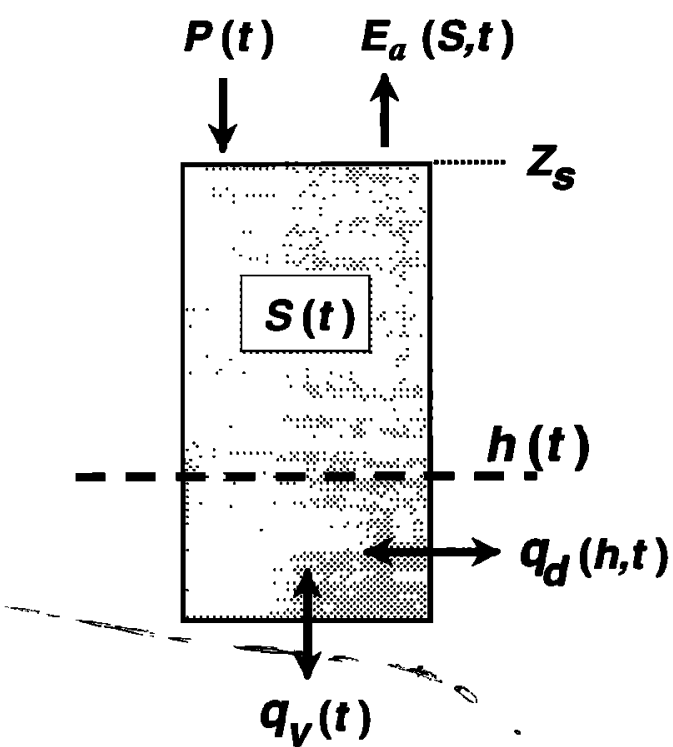

Figure $\neq$ Schematic representation of th model of soilgroundwater interaction.

the hydrological system occur (e.g., adding extra draines). It is based on a stochastic differential equation in order to account for unexplained variability and to be able to quantify the uncertainty of model predictions. Because it explicitly accounts for uncertainty, a stochastic differential equation is a natural tool for design and risk analysis.

This paper is organized as follows. First, a nonlinear stochastic differential equation (SDE) is derived that describes the fluctuation of a shallow water table. Both stationary and nonstationary solutions of the SDE are given. Next, the SDE is embedded in an extended Kalman filter algorithm to calibrate its parameters to measured time series of water table depths. After that, the SDE is calibrated and validated using measurements of groundwater depth from two locations. A section is devoted to using the SDE for design and risk analysis. Finally, in the last section, summary and conclusions are given.

\section{Theoretical Development}

\subsection{A Differential Equation for Water Table Fluctuations}

The purpose of this section is to derive a differential equation that describes water table fluctuations without the need to model flow in the unsaturated zone (i.e., solving Richards' equation). To achieve this, the combined system of soil water and shallow groundwater is modeled using simple mass balance equations. We start with the schematic representation of the system as given in Figure 1. The following variables are depicted:

$h(t)$ height of the phreatic surface at time $t$ relative to some reference level, $L$;

$z_{s}$ surface elevation level relative to some reference level, $L$;

$S(t)$ average saturation of the soil profile at time $t$, which is defined as

$$
S(t)=\frac{1}{z_{s}-h(t)} \int_{h(t)}^{z_{s}}\left[\frac{\theta(z, t)-\theta_{r}}{\theta_{s}-\theta_{r}}\right] d z
$$

$z$ height in soil profile relative to the reference level, $L$;

$\theta(z, t)$ volumetric moisture content at height $z$ and time $t$

$\theta_{s} \quad$ saturated moisture content (constant);

$\theta_{r}$ residual moisture content (constant);

$P(t)$ precipitation rate at time $t, L T^{-1}$;

$E_{a}(S, t)$ actual evapotranspiration flux at time $t, L T^{-1}$.

Actual evapotranspiration is assumed dependent on the average soil saturation following the relation

$$
E_{a}(S, t)=F_{c} E_{p}(t)[S(t)]^{c}
$$

with

$F_{c}$ crop factor (e.g., 1.0 for "standard" grassland [Feddes, 1987]);

$E_{p}(t)$ potential (reference-crop) evapotranspiration flux at time $t, L T^{-1}$, calculated according to Makkink [Winter et al., 1995].

c a constant $(\sim 0.5$ for fully-vegetated soils [Lowry, 1959]);

$q_{v}(t)$ regional exfiltration/infiltration flux from/to deeper groundwater at time $t, L T^{-1}$ (positive in case of exfiltration);

$q_{d}(h, t)$ local flux at time $t$ induced by the presence of surface waters: canals, ditches, drains, and trenches, $L T^{-1}$ (positive in case of drainage).

Usually, several types of surface water systems are present. The total flux $q_{d}(h, t)$ is thought of as being the sum of separate fluxes $q_{d_{i}}(h, t)$ to the $m_{d}$ different water systems present:

$$
q_{d}(h, t)=\sum_{t=1}^{m_{d}} q_{d}(h, t)
$$

Each of these fluxes is dependent on the water table depth:

$$
q_{d_{l}}(h, t)=\frac{h(t)-H_{i}(t)}{\gamma_{l}}
$$

with $H_{i}(t)$ is the drainage base of surface water system $i$ at time $t, L$, and $\gamma_{i}$ is the drainage resistance: resistance to flow of groundwater from/to surface water system $i, T$. For controlled surface waters such as canals and ditches the drainage base equals the controlled surface water level. These surface waters are draining when $h(t)>H_{t}(t)$ and are infiltrating when $h(t)<H_{t}(t)$. The drainage base of a trench and a drain equals the trench bottom elevation and the drain elevation, respectively. Trenches and drains are inactive when $h(t)<H_{t}(t)$.

Forming the mass balance of the combined soil-water and groundwater system results in the following differential equation:

$$
\begin{gathered}
{\left[\varepsilon_{0}+\left(\theta_{s}-\theta_{r}\right)\right] \frac{d h(t)}{d t}+\left(\theta_{s}-\theta_{r}\right) \frac{d\left[\left(z_{s}-h(t)\right) S(t)\right]}{d t}} \\
=P(t)-E_{a}(S, t)+q_{v}(t)-q_{d}(h, t)
\end{gathered}
$$

The first term on the left-hand side represents the groundwater storage change and the second term the storage change in the unsaturated zone. The fluxes are depicted on the right-hand side of the equation. The term $\varepsilon_{0}$ represents an intrinsic storage in the groundwater system that is left when the entire soil profile is saturated. It is added to avoid unstable solutions when the water table is close to ground surface. It can be

where 
thought of as representing storage in macropores and nearby surface depressions.

A solution to (5) is sought in terms of the water table depth only, i.e., without solving Richards' equation. Consequently, we need to find a one-to-one relation between $S(t)$ and $h(t)$, so that $S(t)$ may be replaced by $h(t)$ in (5). This can be achieved by assuming an equilibrium soil moisture profile at all times. This assumption is justified for shallow water table depths, in which case redistribution of soil moisture as a result of precipitation excess or a changing water table will occur rapidly and an equilibrium moisture profile can be expected to occur almost instantaneously. Moreover, the equivalent soil moisture profile, i.e., the soil moisture profile that will allow the time-averaged vertical flux to pass through the soil column, is approximately equal to the equilibrium profile for shallow groundwater tables [Salvucci and Entekhabi, 1994].

If an instantaneous soil moisture equilibrium is assumed at all times, the average soil saturation $S(t)$ is a function of the water table depth only, i.e., $S(t) \equiv S(h(t))$, and the integral of the moisture content in (1) is replaced by an integral of the equilibrium soil moisture profile:

$$
S[h(t)]=\frac{1}{z_{s}-h(t)} \int_{0}^{z_{s}-h(t)}\left[\frac{\theta_{e}(\psi)-\theta_{r}}{\theta_{s}-\theta_{r}}\right] d \psi
$$

where $\psi$ is the suction head (>0), $L$ and $\theta_{e}(\psi)$ is the equilibrium volumetric moisture content for given $\psi$. The equilibrium soil moisture profile $\theta_{e}(\psi)$ is modeled using a Van Genuchtentype relationship [Troch et al., 1993]

$$
\theta_{e}(\psi)=\theta_{r}+\left(\theta_{s}-\theta_{r}\right)\left[\frac{1}{1+(\alpha \psi)^{n}}\right]^{(n+1) / n}
$$

where $\alpha\left[L^{-1}\right]$ and $n$ are Van Genuchten-type parameters. Notice in (7) that instead of the exponent $(n-1 / n)$ that normally appears in Van Genuchten's relationship [Van Genuchten, 1980], the exponent $(n+1 / n)$ is used. This is because with the latter exponent in (7), (6) can be analytically evaluated [Troch, 1992, pp. 144-145]. Troch [1992] also shows that (7) is able to describe experimental soil moisture retention curves with the same accuracy as the original relation of Van Genuchten [1980].

Substitution of (7) into (6) and solving the integral gives the following expression for the average soil saturation for a given water table depth $h(t)$ :

$$
S[h(t)]=\frac{1}{\alpha\left(z_{s}-h(t)\right)}\left\{1+\left[\alpha\left(z_{s}-h(t)\right)\right]^{-n}\right\}^{-1 / n}
$$

From (8) it can be seen that $S(h \rightarrow-\infty)=0$ and that $S\left(h \rightarrow z_{s}\right)=1$.

With (8) for the average soil saturation, the second term on the left-hand side of (5) can be written in terms of the water table depth:

$$
\frac{d\left\{\left[z_{s}-h(t)\right] S[h(t)]\right\}}{d t}=\frac{d}{d h}\left[\left(z_{s}-h\right) S(h)\right] \frac{d h}{d t}
$$

Using (8) in identity (9), one obtains after some manipulation $\frac{d\left[\left(z_{s}-h(t)\right) S(h(t))\right]}{d t}=-\left(\left\{1+\left[\alpha\left(z_{s}-h(t)\right)\right]^{n}\right\}^{-[(n+1) / n]}\right) \frac{d h}{d t}$
Finally, with (10) in (5) the following differential equation for $h(t)$ is obtained

$$
G(h) \frac{d h}{d t}=P(t)-E_{a}[S(h), t]+q_{v}(t)-q_{d}(h, t)
$$

where $S(h)$ is given by (8). The function $G(h)$ represents a specific yield that depends on the water table depth and is given by

$G(h)=\varepsilon_{0}+\left(\theta_{s}-\theta_{r}\right)\left(1-\left\{1+\left[\alpha\left(z_{s}-h\right)\right]^{n}\right\}^{-[(n+1) / n]}\right)$

The limit properties of $G(h)$ are $G(h \rightarrow-\infty)=\varepsilon_{0}+\theta_{s}-$ $\theta_{r}$ and $G\left(h \rightarrow z_{s}\right)=\varepsilon_{0}$, which explains the importance of including the parameter $\varepsilon_{0}$ to obtain stable solutions of (11) for water table depths close to the ground surface.

The derived differential equation for water the table depth (11) is nonlinear for three reasons. First, when the water table rises, more soil moisture is stored in the unsaturated zone, so that the available pore space for groundwater storage $G(h)$ decreases. The result is that the characteristic response time of the water table decreases with a rising water table. Second, the actual evapotranspiration depends on the degree of average soil saturation and thus on the water table depth. Third, the trenches that drain the groundwater when $h(t)>H_{i}(t)$ and are inactive when $h(t)<H_{i}(t)$ also introduce a non-linearity.

\subsection{Stochastic Model Formulation}

In practice, a model like (11) is unable to describe the groundwater fluctuations exactly. Discrepancies will occur between the actual water table depths and those simulated with the model due to errors caused by model assumptions, errors in model parameters, errors in the inputs, and errors in initial conditions. The combined effect of these errors results in uncertain model predictions. This means that a prediction error is made but the value of this error is unknown; at best we know its probability distribution. If the model structure captures the most important dynamics of the water table depth and the model is correctly calibrated, it is reasonable to describe the total prediction error as an additive wideband stochastic process with zero mean [Unny, 1984]. Moreover, as the total error process is the result of a large number of natural and instrumental fluctuations, it can be expected to obey a Gaussian law according to the central limit theorem [cf. Leduc et al., 1986]. Thus a white noise process forms a reasonable approximation for the additive error process. If such a term is added to (11) the following stochastic model is obtained:

$$
G(h) \frac{d h}{d t}=P(t)-E_{a}[S(h), t]+q_{v}(t)-q_{d}(h, t)+\sigma \xi(t)
$$

The process $\xi(t)$ is a white noise process with properties $E[\xi(t)]=0$ and $E\left[\xi\left(t_{1}\right) \xi\left(t_{2}\right)\right]=\delta\left(t_{1}-t_{2}\right)$, where $E$ denotes expectation and $\delta\left(t_{1}-t_{2}\right)$ is the Dirac delta function. The scaling parameter $\sigma\left[L T^{-1}\right]$ determines the magnitude of the white noise process. Because a white noise process is not an ordinary function of time, a solution to (13) cannot be obtained through integration. However, the process $\xi(t)$ can be formally defined as the derivative of a Wiener-Levy process or Brownian motion $\beta(t)$ with independent increments $d \beta_{t}$ in $d t$, which is characterized with $E\left[d \beta_{t}\right]=0$ and $E\left[d \beta_{t}^{2}\right]=d t$ [Unny, 1984]. We then have $\xi(t) d t=\beta(t+d t)-\beta(t) \equiv d \beta_{t}$ so that (13) results in the following stochastic differential equation (SDE) 


$$
d h=a(h, t) d t+\dot{b}(h) d \beta_{t}
$$

with

$$
\begin{gathered}
a(h, t)=\left[\frac{P(t)-E_{a}[S(h), t]+q_{v}(t)-q_{d}(h, t)}{G(h)}\right] \\
b(h)=\left[\frac{\sigma}{G(h)}\right]
\end{gathered}
$$

The general solution of the SDE in (14) is

$$
h\left(t_{1}\right)=h\left(t_{0}\right)+\int_{t_{0}}^{t_{1}} a(h, t) d t+\int_{t_{0}}^{t_{1}} b(h) d \beta_{t}
$$

The second integral on the right-hand side of (16) cannot be solved using ordinary integration methods, because the term $d \beta_{t}$ is of order $\sqrt{d t}$. Instead, stochastic integration must be applied, which is defined in the mean square sense [Jazwinski, 1970; Leduc et al., 1986]. If an SDE describes some physical process, as is the case with (14), it is appropriate to evaluate the stochastic integral in (16) according to Stratonovich [Wong and Zakai, 1965]. Equation (14) is thus interpreted as a Stratonovich-type SDE (as opposed to an Itô-type SDE; see Unny [1984] for an elaborate discussion about the differences between the two types of SDEs.

\subsection{Stationary Solution}

Leduc et al. [1986] give several ways of solving SDEs. One way of obtaining a solution is through direct stochastic integration. While stochastic integration provides closed form solutions for linear SDEs, solutions to nonlinear SDEs are better obtained through the Fokker-Planck equation. In terms of water table depth, the Fokker-Planck equation describes the conditional probability density of $h$ at time $t$, given that $h$ at time $t_{0}$ equals $h_{0}$. If $f_{h}\left(h, t \mid h_{0}, t_{0}\right)$ denotes this conditional probability density function, the Fokker-Planck equation corresponding to the SDE (14) is given by [Gardiner, 1985]

$$
\begin{aligned}
\frac{\partial f_{h}\left(h, t \mid h_{0}, t_{0}\right)}{\partial t}= & -\frac{\partial}{\partial h}\left[a^{*}(h, t) f_{h}\left(h, t \mid h_{0}, t_{0}\right)\right] \\
& +\frac{1}{2} \frac{\partial^{2}}{\partial h^{2}}\left[b^{2}(h, t) f_{h}\left(h, t \mid h_{0}, t_{0}\right)\right]
\end{aligned}
$$

subject to the initial condition

$$
f_{h}\left(h, t_{0} \mid h_{0}, t_{0}\right)=\delta\left(h-h_{0}\right)
$$

and with $a^{*}(h, t)$ defined as

$$
a^{*}(h, t)=a(h, t)+\frac{1}{2} b(h) \frac{\partial b}{\partial h}
$$

The derivative $(\partial b / \partial h)$ in (19), with $b(h)$ defined in (15b), is given in the appendix.

An analytical solution to (17) is possible for the case that $a(h, t) \equiv a(h)$, which entails that the input variables $P(t)$, $E_{p}(t), q_{v}(t)$, and $H_{i}(t)$ are constants. This basically means that the inputs are treated as constants plus stochastic fluctuations that are modeled as wide band noise processes. The sum of these noise processes is subsequently incorporated in the total noise process of (14). A similar approach was followed by Entekhabi et al. [1992] while modeling the large-scale soil moisture variation in the context of climate modeling. While a representation of the inputs as constants with white noise fluc- tuations may be appropriate for the large spatial and temporal scales described by Entekhabi et al. [1992], it is not likely to be a proper representation of the inputs at small temporal scales (days) at a single location. However, as will be shown further on, the stationary solution may be useful for very shallow water tables.

If $a(h, t) \equiv a(h)$, the stochastic process $h(t)$ becomes stationary after some time, and we have $f_{h}\left(h, t \mid h_{0}, t_{0}\right) \equiv$ $f_{h}(h)$, where $f_{h}(h)$ is the stationary probability density function (pdf) of $h(t)$. This also means that the time derivative in the Fokker-Planck equation becomes zero. Consequently, the solution to the Fokker-Planck equation, that is $f_{h}(h)$, follows from solving the following ordinary differential equation:

$$
a^{*}(h) f_{h}(h)=\frac{1}{2} \frac{d}{d h}\left[b^{2}(h) f_{h}(h)\right]
$$

If subsequently the following boundary conditions are adopted $\left(f_{h}(-\infty)=0\right.$ and $f_{h}\left(z_{s}\right)=0$ (reflecting boundaries)), the solution to (20) is [Gardiner, 1985]

$$
f_{h}(h)=\frac{\mathrm{N}}{b^{2}(h)} \exp \left[2 \int_{-\infty}^{h} \frac{a^{*}\left(h^{\prime}\right)}{b^{2}\left(h^{\prime}\right)} d h^{\prime}\right]
$$

where $\mathrm{N}$ is a normalization constant such that

$$
\int_{-\infty}^{z_{s}} f_{h}(h) d h=1
$$

Equation (21) can be evaluated using any type of numerical integration method. Once the stationary pdf $f_{h}(h)$ is known, the pdfs for the average soil saturation $f_{S}(S)$ and the local flux $f_{q_{d}}\left(q_{d}\right)$ can be derived. This is achieved by the derived distribution approach: If $y$ is a monotonous function $g(x)$ of a stochastic variable (or process) $x$ with pdf $f_{x}(x)$, the pdf of $y$ is given by [Papoulis, 1991]

$$
f_{y}(y)=\left|\frac{d g^{-1}(y)}{d y}\right| f_{x}\left[g^{-1}(y)\right]
$$

where $g^{-1}(y)$ denotes the inverse relation of $g(x)$. Using (23) and (3), (4), and (8), the pdfs for soil saturation and the local drainage flux follow from the pdf of water table depth as

$$
\begin{aligned}
& f_{S}(S)=\left[\frac{\left(S^{-n}-1\right)^{1 / n}}{\alpha\left(S^{-n}-1\right) S^{n+1}}\right] f_{h}\left(z_{s}-\frac{\left(S^{-n}-1\right)^{1 / n}}{\alpha}\right) \\
& f_{q_{d}}\left(q_{d}\right)=\left[\sum_{i=1}^{m_{d}} \frac{1}{\gamma_{t}}\right]^{-1} f_{h}\left(\frac{q_{d}+\sum_{t=1}^{m_{d}} \frac{H_{t}}{\gamma_{t}}}{\sum_{i=1}^{m_{d}} \frac{1}{\gamma_{t}}}\right)
\end{aligned}
$$

In (25), only the surface waters are represented that are active for the associated water table depth.

\subsection{Nonstationary Solution}

If the inputs to the SDE (15) are not constant in time, the stochastic process $h(t)$ is nonstationary. If time series of the inputs are available, in principal, it is possible to obtain the pdf $f_{h}\left(h, t \mid h_{0}, t_{0}\right)$ for every $t \geq t_{0}$ by solving the Fokker-Planck equation numerically, for instance, with a finite difference method. Another, much simpler approach is to generate a 
large number of sample functions (or realizations) of the stochastic process $h(t)$ by numerical integration of (14) and then estimating ensemble properties such as $f_{h}\left(h, t \mid h_{0}, t_{0}\right)$ from all of the sample functions; the sample functions of $S(t)$ and $q_{d}(t)$ then follow from $h(t)$ through (3), (4), and (8). An additional advantage of this approach is that not only univariate properties such as the conditional pdf can be estimated, but also multivariate properties such as temporal covariances. Unny [1984] discusses in detail the appropriate methods for numerical integration of SDEs for the generation of sample functions. Here an efficient fourth order Runge-Kutta integration method for Stratonovich-type SDEs was used as given by Newton [1991]. As the inputs are generally available as time series of daily averages, a time step of 1 day was used. Tests on simple SDEs whose sample functions could be analytically calculated showed that using 10 integration steps per time step was sufficient and that 100 integration steps per time step gave excellent results. Therefore it was decided to use 100 integration steps per time step for the generation of sample functions of (14).

\subsection{Calibration of the SDE}

If the SDE is applied at a certain location, the input time series must be collected and the model parameters identified. In the Netherlands, the meteorological input series $P(t)$ and $E_{p}(t)$ can be obtained from the nearest meteorological station (maintained by the Royal Netherlands Meteorological Institute) and the surface water levels $H_{\imath}(t)$ from the local Water Authority. The elevations of trenches and drains with respect to ground surface elevation $z_{s}$ can be obtained from observations around the location. The soil parameters $\theta_{s}, \theta_{r}, \alpha$, and $n$ can be obtained by fitting (7) to an experimental soil moisture retention curve. This curve can be obtained from undisturbed samples taken at the location, but this is usually too expensive. Alternatively, on the basis of a simple description of the local soil profile (i.e., soil texture), a standard retention curve from some Standard Soil Series [e.g., Wösten and Van Genuchten, 1988] can be assigned to the location. The following parameters cannot be obtained from field observations or measurements and therefore must be obtained from calibrating the SDE to measured time series of water table depths: $\varepsilon_{\mathbf{0}}$, $\gamma_{t}\left(i=1, \cdots, m_{d}\right), q_{v}$ (usually assumed constant in time), and $\sigma^{2}$.

Calibration of a deterministic model is usually achieved by comparing model outcomes with measurements and minimizing some least squares criterion. Minimization can be achieved by trial and error, or preferably, by embedding the model in some minimization algorithm. Calibration of a stochastic model is much less straightforward. Instead of one deterministic trace of model outcomes, there are an infinite number of equiprobable sample functions (realizations) that can be compared with measurements. Also, apart from the parameters associated with the deterministic part of the model (i.e., $\varepsilon_{0}, \gamma_{i}$ $\left.\left(i=1, \cdots, m_{d}\right), q_{v}\right)$ also parameters that are associated with the noise process (i.e., $\sigma^{2}$ ) must be calibrated. One way of calibrating a stochastic model to measured data is to embed the model in a discrete-time recursive estimation routine such as a Kalman filter [Sage and Melsa, 1976]. Schweppe [1973] has derived a maximum likelihood criterion in terms of the differences between measurements and predictions made with a Kalman filter. Minimization of this criterion with respect to the parameters to be calibrated (including noise parameters) then leads to unbiased estimates of these parameters.
The maximum likelihood criterion of Schweppe [1973] was developed for linear systems, while we are dealing with a nonlinear SDE. Consequently, some kind of linearization is required; i.e., Schweppe's criterion is used in combination with an extended Kalman filter. The filter equations and the criterion are given hereafter.

The water table depth is described at a finite number of time steps of length $\Delta t$ (in this case: 1 day). Time step $k$ stands for $t=k \Delta t$, so that $h_{k}=h(k \Delta t)$. The following variables are defined:

$\bar{h}_{k}$ the time update, which is the optimal (in least squares sense) linear unbiased estimate of $h_{k}$ at time $k$ using all measurements up to time $k-1$;

$h_{k}$ the measurement update, which is the optimal linear unbiased estimate of $h_{k}$ at time $k$ using all measurements up to time $k$;

$\sigma_{k \mid k-1}^{2}$ variance of the error in the time update: $\operatorname{var}\left[h_{k}-\bar{h}_{k}\right]$;

$\sigma_{k \mid k}^{2} \quad$ variance of the error in the measurement update: var $\left[h_{k}-h_{k}\right]$;

$y_{k}$ measurement of $h_{k}$ at time $k$;

$\sigma_{y_{k}}^{2}$ variance of the measurement error.

The time update can be obtained by using the Runge-Kutta algorithm [Newton, 1991] to evaluate the deterministic part of the SDE, with the measurement update of the previous time step as initial condition:

$$
\bar{h}_{k}=h_{k-1}+\int_{(k-1) \Delta t}^{k \Delta t} a(h, t) d t
$$

To obtain an expression for the variance of the error in the time update we first have to discretize the SDE in (14):

$$
h_{k}=\phi\left(h_{k-1}, k-1\right)+b\left(h_{k-1}\right) \Delta \beta_{k}
$$

where

$$
\phi\left(h_{k}, k\right)=h_{k}+a\left(h_{k}, k\right) \Delta t
$$

and $\Delta \beta_{k}$ is the increment of the Brownian motion process $\beta(t)$ during the time interval $[(k-1) \Delta t, k \Delta T]$ with variance equal to $\Delta t^{\prime}$ (in dimensionless time; i.e., if we decided to work with the unit "hours" and we used a time step of 3 hours, we would have $\Delta t^{\prime}=3$ ). Equation (27) is a nonlinear discrete time state equation. To assess the uncertainty in the time update (26), (27) is linearized around the measurement update at the previous time step as follows [Schweppe, 1973, p. 406]

$$
\begin{aligned}
h_{k}= & \phi\left(\hat{h}_{k-1}, k-1\right)+\Phi\left(\hat{h}_{k-1}, k-1\right)\left[h_{k-1}-\hat{h}_{k-1}\right] \\
& +b\left(h_{k-1}\right) \Delta \beta_{k}
\end{aligned}
$$

with

$\Phi\left(\hat{h}_{k-1}, k-1\right)=\left.\frac{\partial \phi}{\partial h}\right|_{h=\hat{h}_{k-1}}=1+\Delta t\left[\left.\frac{\partial a}{\partial h}\right|_{h=\hat{h}_{k-1}}\right]$

where the derivative $(\partial a / \partial h)$ is given in the appendix.

As we have from (26) and (28) that $\phi\left(\hat{h}_{k-1}, k-1\right) \approx \bar{h}_{k}$, we obtain from (29)

$h_{k}-\bar{h}_{k}=\Phi\left(\hat{h}_{k-1}, k-1\right)\left[h_{k-1}-\hat{h}_{k-1}\right]+b\left(\hat{h}_{k-1}\right) \Delta \beta_{k}$

Finally, from (31) the variance of the error in the time update can be approximated with 
(a)

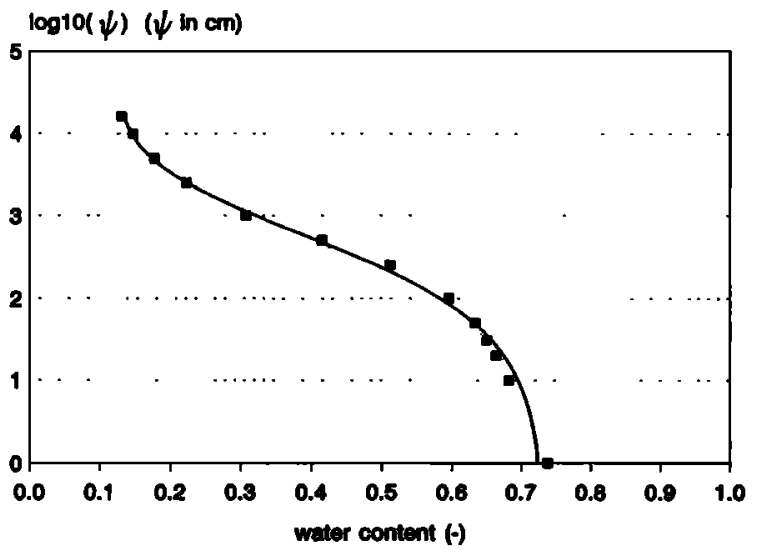

(b)

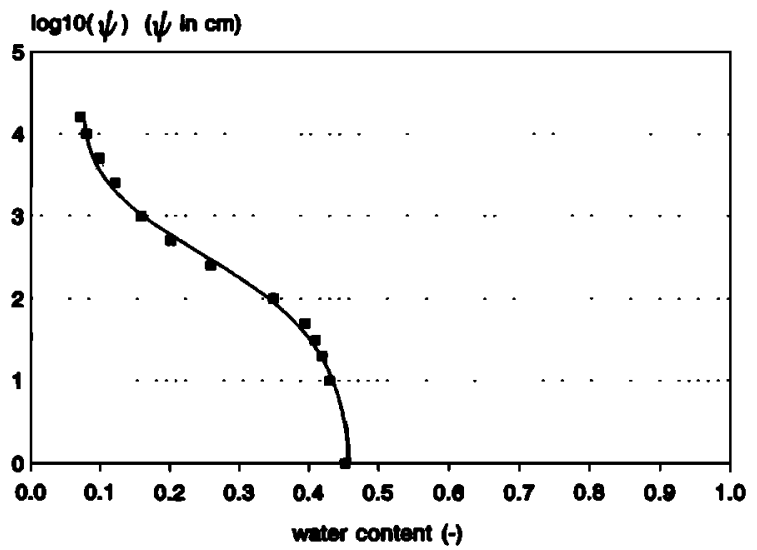

Figure 2. Fit of (7) (solid line) through standard soil moisture retention data (squares) from the Standard Soil Series of the Netherlands [Wösten and Van Genuchten, 1988]: (a) location 12el0003 and (b) location $12 \mathrm{bl0015.}$

$$
\sigma_{k \mid k-1}^{2}=\Phi^{2}\left(h_{k-1}, k-1\right) \sigma_{k-1 \mid k-1}^{2}+b^{2}\left(\hat{h}_{k-1}\right) \Delta t^{\prime}
$$

The measurement update and the associated error variance can be calculated using the following equations: (1) if at time step $k$ a measurement is taken,

$$
\begin{gathered}
n_{k}=y_{k}-\bar{h}_{k} \\
\sigma_{n_{k}}^{2}=\sigma_{k \mid k-1}^{2}+\sigma_{y_{k}}^{2} \\
K_{k}=\sigma_{k \mid k-1}^{2}\left(\sigma_{n_{k}}^{2}\right)^{-1} \\
\hat{h}_{k}=\bar{h}_{k}+K_{k} n_{k} \\
\sigma_{k \mid k}^{2}=\left(1-K_{k}\right) \sigma_{k \mid k-1}^{2}
\end{gathered}
$$

(2) if at time step $k$ no measurement is taken,

$$
\begin{gathered}
\hat{h}_{k}=\bar{h}_{k} \\
\sigma_{k \mid k}^{2}=\sigma_{k \mid k-1}^{2}
\end{gathered}
$$

The variable $K_{k}$ is the Kalman gain, $n_{k}$ is called the innovation $[L]$, and $\sigma_{n_{k}}^{2}$ the innovation variance $\left[L^{2}\right]$. The complete extended Kalman filter algorithm is formed by the recursive application of (26) and (32) through (39) with the initial conditions $h_{0}$ and $\sigma_{0 \mid 0}^{2}$.

Suppose that the Kalman filter is run for a calibration period with $N$ measurements using a given vector of parameters $\boldsymbol{\mu}^{T}=$ $\left(\varepsilon_{0}, \gamma_{1}, \cdots, \gamma_{m_{d}}, q_{v}, \sigma^{2}\right)$. The corresponding innovations and innovation variances $n_{k}(\mu), \sigma_{n_{k}}^{2}(\mu),(k=1, \cdots, N)$ constitute the following criterion:

$J(N ; \boldsymbol{\mu})=N \ln (2 \pi)+\sum_{k=1}^{N} \sigma_{n_{k}}^{2}(\boldsymbol{\mu})+\sum_{k=1}^{N}\left[\frac{n_{k}^{2}(\boldsymbol{\mu})}{\sigma_{n_{k}}^{2}(\boldsymbol{\mu})}\right]$

Equation (40) equals minus one-half times the log-likelihood function that can be derived for innovations of a Kalman filter, provided that the state equation is linear and both the noise $\Delta \boldsymbol{\beta}_{k}$ and the measurement error are Gaussian distributed [Schweppe, 1973; te Stroet, 1995]. In that case, minimization of (40) with respect to the parameters $\boldsymbol{\mu}$ provides a maximum likelihood estimate of the parameters. Here the criterion is used in combination with a nonlinear state equation. As a consequence, (40) is only an approximation of the loglikelihood function and will not provide exact maximum likelihood estimates. However, a simulation study has shown that despite this, good estimates of the parameters can be obtained (M. F. P. Bierkens, unpublished results, 1997).

The extended Kalman filter and Schweppe's criterion have been implemented in a calibration program. Minimization of the object function (40) is performed with the downhill simplex method as given by Press et al. [1986]. This method was chosen because it does not require derivatives of the object function.

\section{Application at Two Locations}

\subsection{Description of the Data}

The SDE was applied at two locations in the north of the Netherlands. At both locations, water table depths have been recorded with a frequency of 2 times a month for a period of at least 10 years. The period 1982-1991 (10 years) was selected to serve as calibration and validation period. Daily averaged precipitation and potential evapotranspiration rates were available for the period 1962-1991, recorded at the nearby meteorological station Eelde by the Royal Netherlands Meteorological Institute. At both locations, descriptions of the soil profile have been made [Knotters and Van Walsum, 1997]. The first location, which is coded $12 \mathrm{el} 0003$ consists of a peat soil of $100 \mathrm{~cm}$ on top of medium fine sand. Water table depths are very shallow here, varying between 0.0 and $0.9 \mathrm{~m}$ below ground surface. The second location is termed $12 \mathrm{bl0015}$ and consists entirely of loamy fine sand. At this location, water table depths are slightly deeper, varying between 0.2 and $1.5 \mathrm{~m}$ below ground surface. Standard soil moisture retention data for peat and loamy fine sand were taken from the Standard Soil Series of the Netherlands [Wösten and Van Genuchten, 1988] and assigned to the locations $12 \mathrm{el0003}$ and $12 \mathrm{bl0015}$, respectively. The soil parameters were obtained by a least squares fit of (7) to the standard soil moisture retention data. Figure 2 shows that (7) fits these data very well.

The land cover at both locations consists of grass, which means that the crop factor $F_{c}=1.0$ [Feddes, 1987]. The exponent $c$ (equation (2)) is taken as 0.5 according to a fully vegetated situation [Lowry, 1959]. At both locations a ditch 

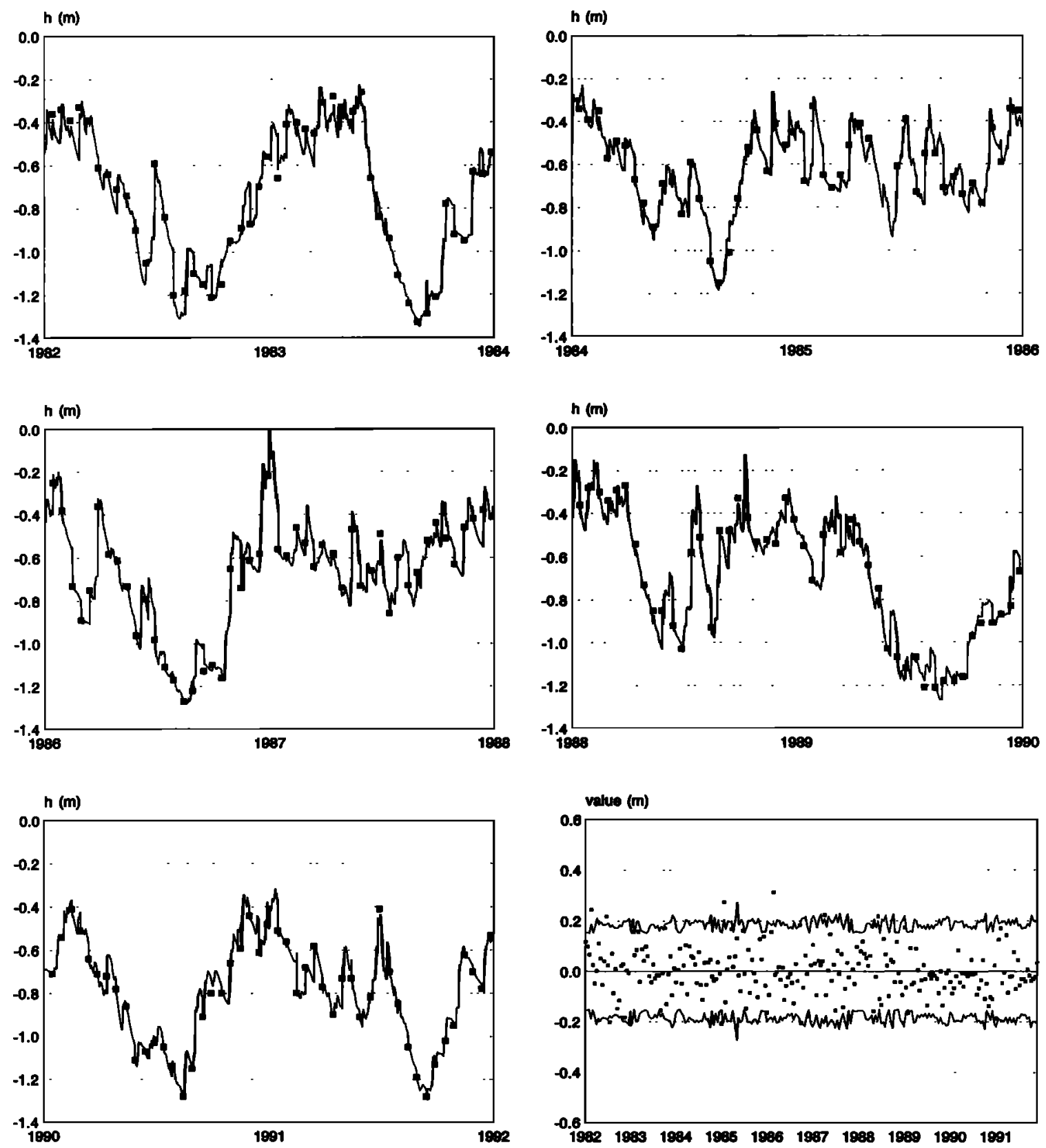

Figure 3. Time updates (solid line) of the extended Kalman filter and measurements (squares) at location 12bl0015; SDE calibrated on period 1982-1991; bottom right: innovations (squares) and 2.5 and 97.5 percentiles (solid line) of the innovations.

with a controlled water level is present, as well as a trench that is only active for water table elevations higher than its bottom elevation. Knotters and Van Walsum [1997] measured the surface water levels of the ditches and the bottoms of the trenches relative to the surface elevation at the locations. For both locations, Table 1 gives the values for the parameters that have been determined from the profile descriptions and field observations and need not be calibrated. Notice that the surface elevation $z_{s}$ is taken as reference level at both locations $\left(z_{s}=0\right)$.

\subsection{Calibration, Validation, and Verification}

The available measurements of water table depth (19821991) for both locations were divided into two periods: (19821986) and (1987-1991). The SDE was calibrated to each of these periods separately, as well as to the complete 10-year period. The calibrated parameter values for both locations are shown in Table 2. It can be seen that for $12 \mathrm{bl0015}$ similar parameter values are obtained for all three periods. The results for location $12 \mathrm{el} 0003$ show that the values of $q_{v}$ and $\sigma^{2}$ are different for the three periods. Apparently, some nonstationarity is present in the series of water table depths that is not accounted for by the model. Inquiry to the abstraction rates of the nearby pumping station "Onnen" showed an average abstraction rate of $9.6 \times 10^{6} \mathrm{~m}^{3} / \mathrm{yr}$ for the period $1982-1986$ and an average abstraction rate of $10.6 \times 10^{6} \mathrm{~m}^{3} /$ year for the period 1987-1991. This may partly explain the larger $q_{v}$ in the latter period. Other possible reasons may be intensified drainage or persistent long-term meteorological fluctuations that 
Table 1. Parameters at Locations $12 \mathrm{el} 0003$ and $12 \mathrm{bl0015}$ Obtained From Profile Descriptions and Field Observations i.e., Parameters That Are Not Calibrated

\begin{tabular}{lll}
\hline & $12 \mathrm{el} 0003$ & $12 \mathrm{~b} 10015$ \\
\hline$c$ & 0.5 & 0.5 \\
$F_{c}$ & 1.0 & 1.0 \\
$\theta_{s}$ & 0.73 & 0.47 \\
$\theta_{r}$ & 0.12 & 0.073 \\
$\alpha, \mathrm{cm}^{-1}$ & 0.000434 & 0.000785 \\
$n$ & 0.677 & 0.701 \\
$z_{s}, \mathrm{~m}$ & 0.0 & 0.0 \\
$H_{1}, \mathrm{~m}$ & -0.3 & -0.8 \\
$H_{2}, \mathrm{~m}$ & -0.2 & -0.5 \\
\hline
\end{tabular}

influence the regional groundwater flow. The larger $\sigma^{2}$ can be expected for deeper water tables, which are less damped by ditches and trenches and thus show more variation in time.

For illustration, Figure 3 shows for location $12 \mathrm{bl0015}$ the Kalman filter time updates together with the measurements, after the SDE is calibrated to the entire period. The bottom right portion of Figure 3 shows the innovations, together with the 2.5- and 97.5 percentiles of the innovations calculated with the Kalman filter $\left( \pm 2 \sigma_{n_{k}}\right)$. The fraction innovations outside these limits equals 0.042 (it should be 0.05 ). For location $12 \mathrm{e} 10003$ this fraction equals 0.046 .

To validate the deterministic part of the SDE separately, the following steps were taken: The SDE that was calibrated on period 1982-1986 was run for the period 1982-1991 while setting $\sigma^{2}$ equal to 0 . The resulting (deterministic) time series of predictions was compared with the measurements of period 1982-1986 (verification) and the measurements of period 1987-1991 (validation). This was repeated for the SDE calibrated on the period 1987-1991 and the SDE calibrated on period 1982-1991, where in the latter case comparison of model predictions and measurements involves the whole period (i.e., verification only). The following statistics of measured and predicted water table depths were calculated:

Mean error

$$
\mathrm{ME}=\frac{1}{N} \sum_{t=1}^{N}\left(y_{t}-h_{t}\right)
$$

Root mean square error

$$
\mathrm{RMSE}=\sqrt{\frac{1}{N} \sum_{t=1}^{N}\left(y_{t}-\breve{h}_{\imath}\right)^{2}}
$$

Table 2. Calibrated Parameters at Locations 12 el0003 and 12 bl0015 for Different Periods

\begin{tabular}{cccccc}
\hline Period & $\varepsilon_{0}$ & $\begin{array}{c}\gamma_{1}, \\
\text { days }\end{array}$ & $\begin{array}{c}\gamma_{2}, \\
\text { days }\end{array}$ & $\begin{array}{c}q_{w}, \\
\mathrm{~mm} / \mathrm{d}\end{array}$ & $\begin{array}{c}\sigma^{2}, \\
\mathrm{~mm}^{2} / \mathrm{d}^{2}\end{array}$ \\
\hline \multicolumn{7}{c}{12 el0003 } \\
$1982-1986$ & 0.023 & 112.57 & 20.15 & -1.85 & 22.89 \\
$1987-1991$ & 0.063 & 113.94 & 19.30 & -2.58 & 40.70 \\
$1982-1991$ & 0.047 & 112.86 & 22.58 & -2.26 & 34.63 \\
$1982-1986$ & 0.063 & 391.06 & 92.40 & -0.21 & 25.45 \\
$1987-1991$ & 0.058 & 328.89 & 91.68 & -0.32 & 19.81 \\
$1982-1991$ & 0.066 & 369.59 & 88.37 & -0.24 & 23.28 \\
\hline
\end{tabular}

\begin{tabular}{|c|c|c|c|c|c|c|c|c|c|}
\hline & \multicolumn{3}{|c|}{ 1982-1986 } & \multicolumn{3}{|c|}{$1987-1991$} & \multicolumn{3}{|c|}{$1982-1991$} \\
\hline & ME & RMSE & $p$ & ME & RMSE & $p$ & ME & RMSE & $p$ \\
\hline \multicolumn{10}{|c|}{$12 \mathrm{el} 0003$} \\
\hline 1 & -0.006 & 0.108 & 0.06 & -0.080 & 0.146 & 0.06 & & & \\
\hline 2 & 0.066 & 0.128 & 0.15 & -0.008 & 0.112 & 0.04 & & & \\
\hline 3 & & & & & & & -0.005 & 0.117 & 0.05 \\
\hline \multicolumn{10}{|c|}{$12 b l 0015$} \\
\hline 1 & -0.009 & 0.121 & 0.03 & -0.031 & 0.109 & 0.03 & & & \\
\hline 2 & 0.011 & 0.122 & 0.05 & -0.010 & 0.104 & 0.04 & & & \\
\hline 3 & & & & & & & -0.016 & 0.114 & 0.04 \\
\hline
\end{tabular}

Table 3. Validation and Verification Results

ME, mean error, meters; RMSE, root mean square error, meters; $p$, fraction of residuals outside 2.5 and 97.5 percentiles; 1 , calibrated on 1982-1986; 2, calibrated on 1987-1991; 3, calibrated on 1982-1991.

where $N$ is the number of measurements, $y_{\imath}$ the $i$ th measurement and $\breve{h}_{i}$ the prediction of the deterministic model at the same time as the measurement was taken. The difference $y_{i}-\bar{h}_{i}$ is called a residual here to distinguish it from the innovation $y_{t}-\bar{h}_{t}$ that is obtained from the extended Kalman filter, in which case the predictions are updated regularly with measurements. The variance of $y_{1}-\breve{h}_{t}$ can, however, be calculated with the extended Kalman filter by running it without measurement updates (equations (26), (32), (38), and (39)). Its value in this case is given by $\sigma_{k \mid k-1}^{2}$. The 2.5 - and 97.5-percentiles of the residuals are then approximated with $\pm 2 \sigma_{k \mid k-1}$. As with the innovations, we can calculate the fraction of residuals outside the 2.5 - and 97.5 percentiles and see if it is close to 0.05 . If this is the case, the prediction errors are effectively modeled with (32) and $\sigma^{2}$ has the correct value.

The validation and verification results are summarized in Table 3. The assumed nonstationarity that is not captured by the model can be seen in the ME of location 12el0003 for both 5-year periods: a model that is calibrated on the period 19821986 overestimates the water table depth in the period 1987$1991(\mathrm{ME}=-0.080 \mathrm{~m})$ and vice versa $(\mathrm{ME}=0.066 \mathrm{~m})$. The large fraction of residuals outside the 2.5 and 97.5 percentiles (0.15) can also be attributed to this nonstationarity. The validation and verification results for location $12 \mathrm{bl0015}$ are satisfactory for all periods.

Knotters and Van Walsum [1997] applied transfer functionnoise models (TFN) and the soil-moisture accounting model SWATRE at the same locations. The TFN models used by Knotters and Van Walsum [1997] predict time series of water table depth through a linear relation between the precipitation excess $P(t)-E_{p}(t)$ and the water table depth. Using SWATRE [Belmans et al., 1983], time series of water table depth are predicted by solving Richards' equation for unsaturated flow in heterogeneous soils. These models were cali-

Table 4. Comparison of Validation Results of Predictions With the SDE and Predictions of a Transfer Function Noise Model (TFN) and the Soil-Moisture Accounting Model SWATRE for the Period 1982-1986

\begin{tabular}{ccccccccc}
\hline & \multicolumn{2}{c}{ SDE } & & \multicolumn{2}{c}{ TFN } & & \multicolumn{2}{c}{ SWATRE } \\
\cline { 2 - 3 } \cline { 8 - 9 } & ME & RMSE & & ME & RMSE & & ME & RMSE \\
\hline 12el0003 & 0.066 & 0.128 & & 0.068 & 0.137 & & 0.065 & 0.132 \\
12bl0015 & 0.011 & 0.122 & & 0.021 & 0.126 & & 0.036 & 0.128 \\
\hline
\end{tabular}



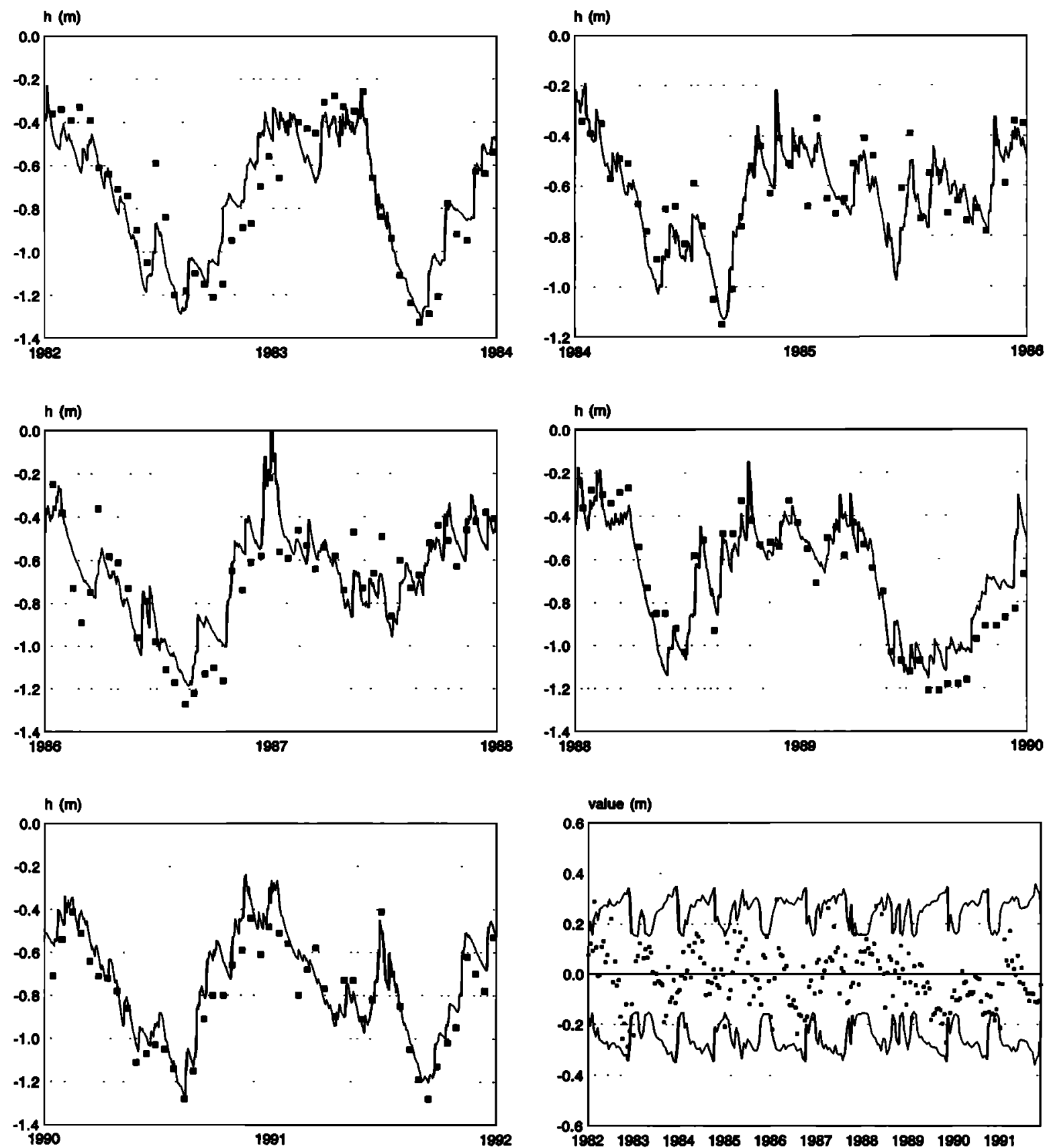

Figure 4. Predictions with the deterministic model (solid line) and measurements (squares) at location $12 \mathrm{bl0015}$; SDE calibrated on period 1982-1991; bottom right: residuals (squares) and 2.5 and 97.5 percentiles (solid line) of the residuals.

brated at locations $12 \mathrm{el} 0003$ and $12 \mathrm{~b} 10015$ using the measured water tables from 1987 to 1991. In Table 4 the results of validating the predictions of these models on the period 1982 1986 are compared with the validation results of the SDE. These results show that the SDE performs equally well or slightly better than the TFN model and SWATRE.

Figure 4 shows for location $12 \mathrm{bl0015}$ the predictions with the deterministic model together with the measurements. Also shown are the residuals and their 2.5 and 97.5 percentiles. There is no one-to-one relation between the width of this interval and the water table depth because there are two mechanisms that work in opposite directions. This can be seen from (32). When the water table is falling, the influence of the additional noise (through $b\left(h_{k}\right)$ ) decreases. However, because of increasing specific yield and particularly because the trenches become nonactive, $\Phi\left(h_{k}, k\right)$ increases. This means that the memory of the system increases and prediction errors will propagate further in time, resulting in a larger prediction error. The net effect is that the prediction error increases during the falling limb of the water table. It increases even stronger during the rising limb because apart from the effects of error propagation, the influence of the noise term through $b\left(h_{k}\right)$ increases. When the rising water table reaches a certain depth, $\Phi\left(h_{k}, k\right)$ decreases drastically, partly because of the specific form of the soil moisture retention curve for $\psi$ close to 0 , but more important because the trenches start to drain the 
Table 5. Comparison of Univariate Statistics of Water Table Depths for the Period 1982-1991

\begin{tabular}{lccc}
\hline & Meas. & Sim. & Stat. \\
\hline & 12 el0003 & & \\
Mean & -0.484 & -0.487 & -0.477 \\
Standard deviation & 0.183 & 0.178 & 0.139 \\
Third central moment & 0.00190 & 0.000661 & 0.000179 \\
P25 & -0.64 & -0.613 & -0.573 \\
P50 & -0.51 & -0.484 & -0.480 \\
P75 & -0.33 & -0.356 & -0.384 \\
MHW & -0.202 & -0.212 & $\ldots$ \\
MLW & -0.712 & -0.764 & $\ldots$ \\
& $12 b l 0015$ & & \\
Mean & -0.706 & -0.712 & -0.659 \\
Standard deviation & 0.270 & 0.256 & 0.175 \\
Third central moment & -0.00833 & -0.0123 & -.00163 \\
P25 & -0.90 & -0.901 & -0.777 \\
P50 & -0.67 & -0.677 & -0.649 \\
P75 & -0.51 & -0.499 & -0.530 \\
MHW & -0.367 & -0.346 & $\ldots$ \\
MLW & -1.085 & -1.103 & $\ldots$ \\
\hline
\end{tabular}

Depths are in meters. Here meas., estimated from the measurements; sim., estimated from 1000 simulated sample functions; stat., calculated with the stationary solution (21).

groundwater. This results in a sudden decrease of the memory of the system and thus of the prediction error.

\subsection{Reproduction of Univariate Statistics}

With the SDE calibrated on the period 1982-1991, 1000 sample functions of daily water table depth for this period were simulated, using (14) and the Runge-Kutta algorithm of Newton [1991]. Univariate statistics were estimated from these simulations and compared with those estimated from the data. Bivariate statistics such as covariances were not considered because the process $h(t)$ is nonstationary. Moreover, as only semimonthly measurements are available, covariances for smaller lags cannot be estimated.

The following statistics were calculated: mean, standard deviation, third central moment, and the 25,50 , and 75 percentiles. Furthermore, two additional statistics were calculated that are frequently used in the Netherlands to characterize the fluctuation range of the water table: the mean highest water table (MHW) and the mean lowest water table (MLW) [Van der Sluijs and De Gruijter, 1985]. They are calculated by taking for each "hydrological" year (starting April 1 and ending on March 31 of the next year) the three highest and three lowest water table depths observed. If measurements have been taken for $M$ consecutive hydrological years, then the MHW for this period is estimated by averaging the $3 M$ highest water tables depths observed. Similarly, the MLW follows from averaging the $3 M$ lowest water table depths.

It was also checked how well the statistics of the pdf given by the stationary solution in (21) compare with those estimated from the measurements (recall that stationary solution assumed the input process to be a constant plus white noise). In order to apply the stationary solution, the variance of the net nonsteady inputs (precipitation - potential evapotranspiration) is added to $\sigma^{2}$. So the total noise now consists of the sum of an error process and the input process where both are assumed to be white noise. The variance of $P(t)-E_{p}(t)$ can be estimated from the meteorological time series of the station Eelde and equals $18.77 \mathrm{~mm}^{2} / \mathrm{d}^{2}$. Adding this to the $\sigma^{2}$ as calibrated for the period 1982-1991 gives a total noise variance of $53.40 \mathrm{~mm}^{2} / \mathrm{d}^{2}$ for location $12 \mathrm{el0003}$ and $42.05 \mathrm{~mm}^{2} / \mathrm{d}^{2}$ for location $12 \mathrm{bl0015}$.

In Table 5 for both locations the statistics estimated from the measurements are compared with those estimated from the simulations and those calculated with the stationary solution. Of course, the stationary solution only gives a pdf and not the MHW and MLW. At this point it must be noted that the statistics in Table 5 are estimated from all the simulated daily water table depths (averages over time and realizations). Consequently, they are univariate ensemble statistics of the stochastic process $h(t)$, representing both uncertainty and variation with time simultaneously.

A frequency of exceedance graph (FOE graph) is one way of representing the cumulative probability of water table depth. This graph gives the expected number of days per year that the water table depth exceeds a certain level. Figures 5 and 6 show for both locations the FOE graphs as estimated from the measurements, together with those estimated from the 1000 simulations and the ones obtained from the stationary pdf. As with (a)

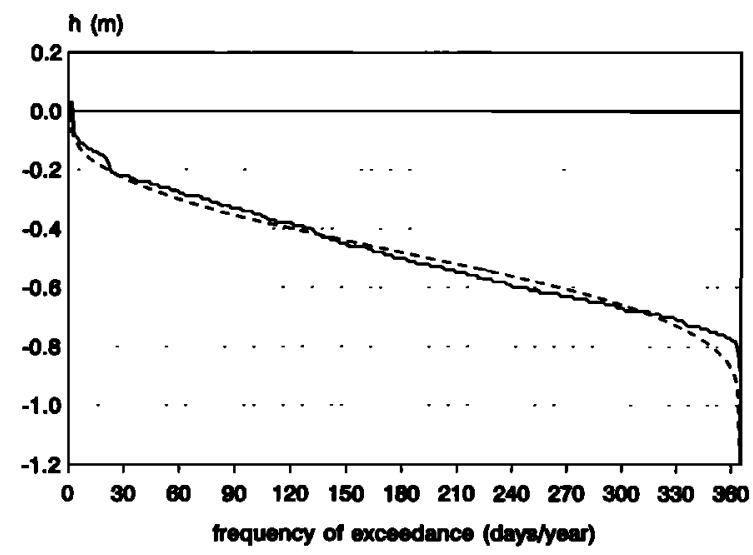

(b)

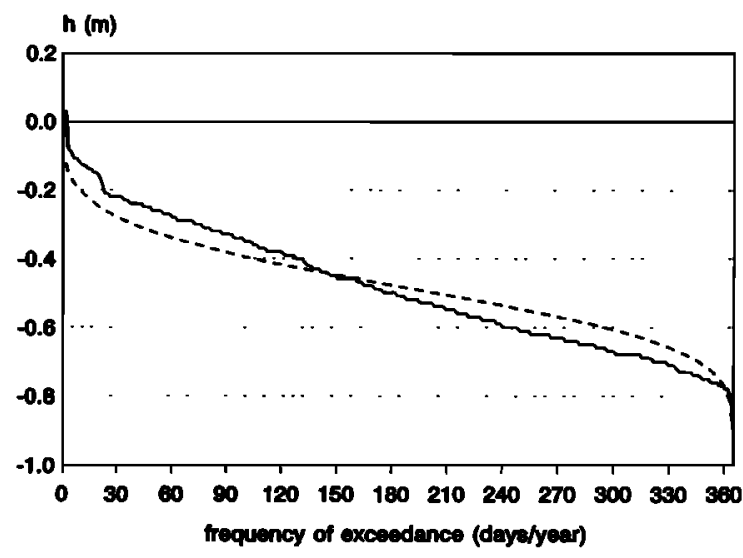

Figure 5. Frequency of exceedance of water table depth at location 12el0003 for the period 1982-1991; (a) estimated from measurements (solid line) and estimated from 1000 simulated sample functions (dashed line); (b) estimated from measurements (solid line) and calculated with the stationary solution of (21) (dashed line). 
(a)

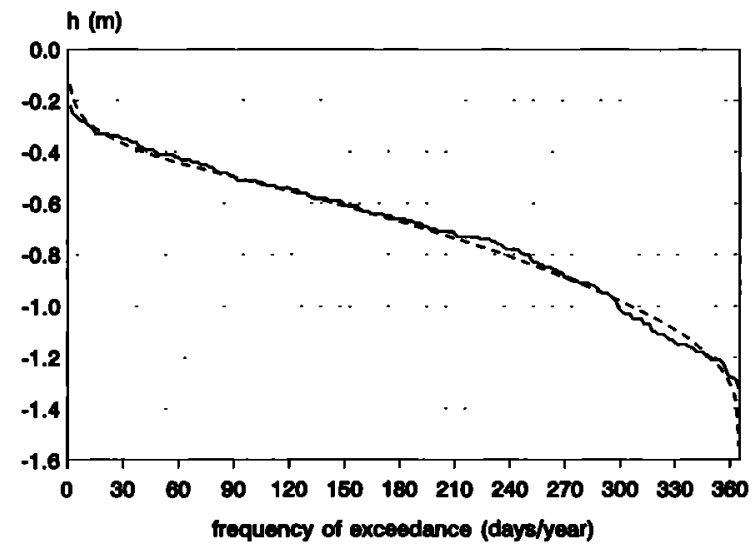

(b)

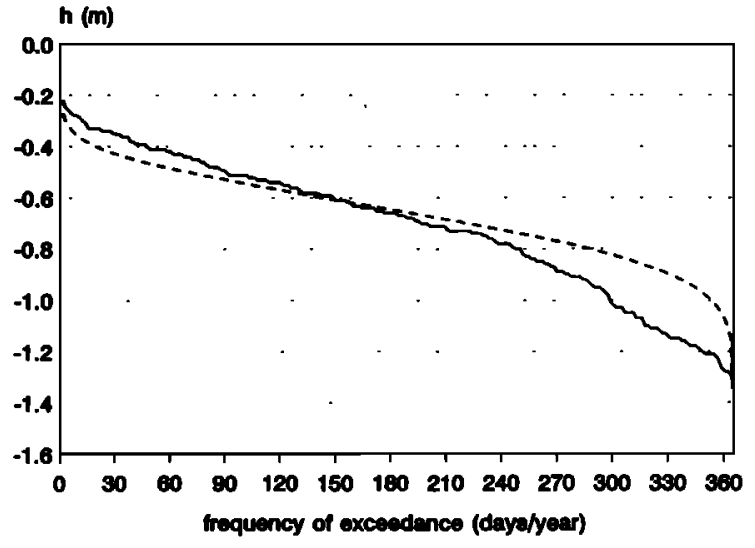

Figure 6. Frequency of exceedance of water table depth at location 12bl0015 for the period 1982-1991; (a) estimated from measurements (solid line) and estimated from 1000 simulated sample functions (dashed line); (b) estimated from measurements (solid line) and calculated with the stationary solution of (21) (dashed line).

the statistics, the FOE graph obtained from the simulations represents both the natural variation and the uncertainty about $h(t)$.

Table 5 and Figures 5 and 6 show that the univariate statistics of the water table depth are well reproduced by the 1000 simulated sample functions, especially for location $12 \mathrm{bl} 10015$. The stationary solution performs poorly for location $12 \mathrm{bl} 0015$. It underestimates the variation of the water table depth by neglecting the temporal correlation in the meteorological inputs. However, for location $12 \mathrm{el0003}$ the stationary solution performs almost as well as the $\mathbf{1 0 0 0}$ simulations. An explanation for this must be sought in the characteristic response time of the soil-groundwater systems at the two locations. In analogy with the step response of a linear system, some characteristic response time of a non-linear system can be obtained by observing how fast it tends to an equilibrium state. This is shown in Figure 7. The water table for the SDEs at both locations is set to $-1.0 \mathrm{~m}$. Then, using only the deterministic part of the SDE, the reaction to a constant input of $P$ and $E_{p}$, here the averages 2.09 and $1.41 \mathrm{~mm} / \mathrm{d}$, respectively, is observed. From Figure 7 it can be seen that the system at location 12 bl0015 takes $\sim 3$ times longer to reach an equilibrium state

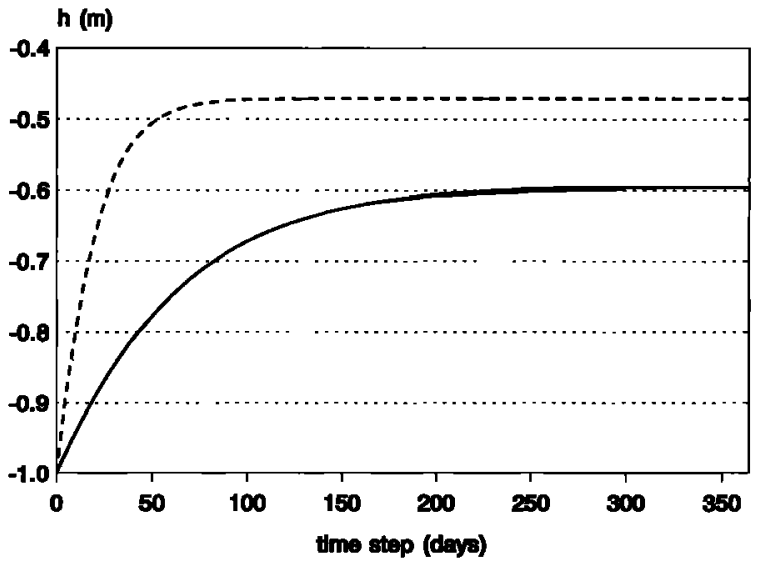

Figure 7. Illustration of the characteristic response times for the water table depth at location $12 \mathrm{el0003}$ (dashed line) and $12 \mathrm{bl0015}$ (solid line). than that of location $12 \mathrm{el} 0003$. If a system reacts more slowly, such as that of location 12bl0015, an input series of persistent positive or persistent negative net inputs is required if this system is to traverse its possible range of variation. This is not likely to occur when the inputs obey a white noise process. Hence the stationary solution will underestimate the actual range of variation, as can be seen from the standard deviation in Table 5 and from the FOE graph in Figure 6b. In contrast, a fast reacting system will reach the boundaries of its range of variation, even if the net inputs are positive or negative for just a few time steps. So it can be expected that the stationary solution works well for fast reacting systems, i.e., locations with very shallow water tables and an intensive drainage system.

\subsection{Extrapolation for Design and Risk Analysis}

Measurements of water table depths that are taken over a short period of time (several years) reflect the weather conditions that prevailed during the observation period. For instance, in the Netherlands the second half of the 1980s represents a relatively dry period. Drainage systems that are designed, on the basis of water table depths that were observed during this period only, are likely to be too small in wetter periods. Ideally, the drainage system, including weirs and pumping stations, must be designed for the weather conditions over a period of $\mathbf{3 0}$ years or more. Once calibrated on measurements of water table depths taken over a limited period of time, the SDE can be used to generate sample functions of a considerably longer period, provided that meteorological data for this longer period are available (see Knotters and Van Walsum [1997] for an earlier application of this principle). This is generally the case, as the Royal Netherlands Meteorological Institute has been collecting meteorological data on a daily basis at a large number of locations since the 1950s. The generated sample functions of water table depth, and if required those of average soil saturation and the local drainage flux, can be used as a basis for the design of drainage systems and the management of such systems. Also, the effects of changing the drainage system or its management and the effects of a different regional flux or changing climate conditions can be readily analyzed using simulations with the SDE. Because a large number of sample functions can be analyzed, the 
(a)

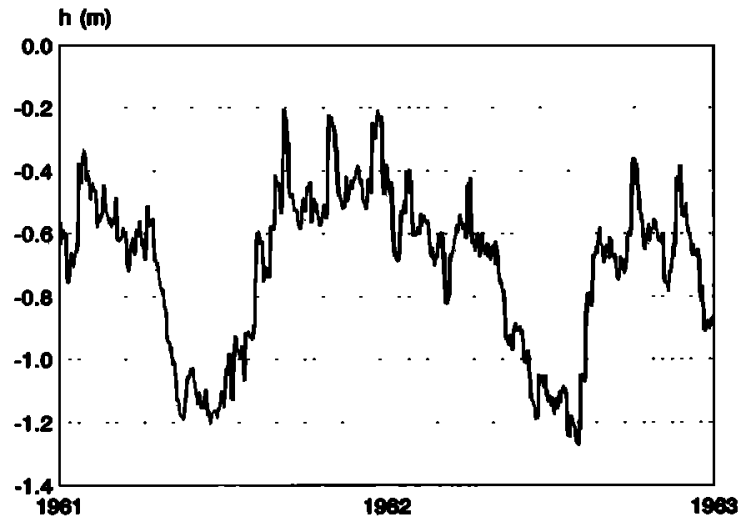

(b)

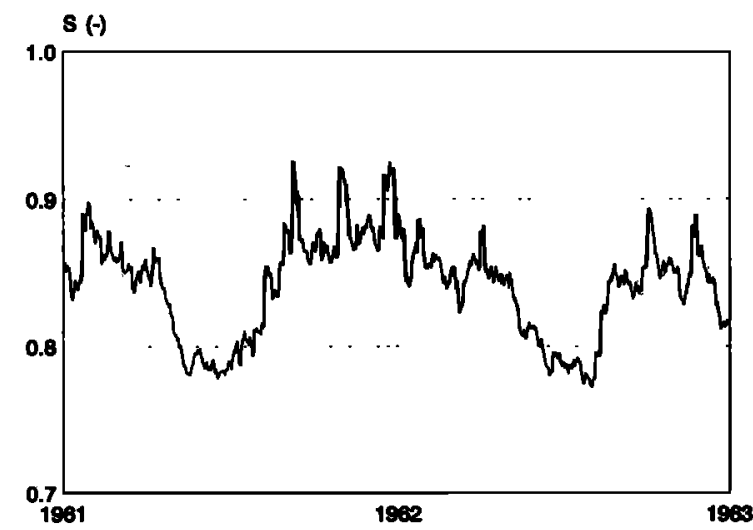

(c)

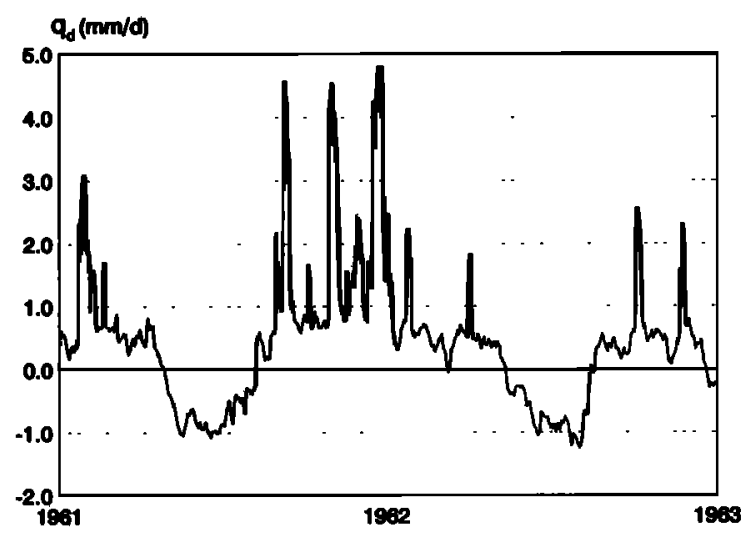

Figure 8. Parts of a sample function simulated at location 12 bl0015 for the period 1962-1991: (a) water table depth, (b) average soil saturation, and (c) local flux from/to surface waters.

design process can be supplemented with uncertainty measures which may serve as the basis for a risk analysis.

As an example, 500 sample functions of 30 years of daily water table depths, soil saturation and local drainage flux were simulated for location 12b10015. The parameters used were those obtained from calibrating the SDE on measurements of water table depth from the period 1982-1991. For the 30 year simulations time series of daily precipitation and potential evapotranspiration from 1962 to 1991 were used as inputs. Figure 8 shows the first 2 years of a simulated sample function of water table depth and the associated average soil saturation and local drainage flux.

Because the period 1982-1991 contained both wet and dry years, the univariate statistics calculated from the 500 30-year sample functions are very similar to those of Table 5 and are therefore not given here. Instead, Figure 9 shows three examples of measures that describe the regime of water table depth at location 12bl0015, including both natural variation and uncertainty. In Figure 9a, for every day number, the average value, the median value and the 2.5 and 97.5 percentiles of water table depth are given. Such curves thus provide for a given day the probability distribution of water table depth. Figure $9 \mathrm{~b}$ shows the expected number of consecutive days per year that the water table depth exceeds a certain level. In Figure 9c, duration of exceedance graphs are depicted. For instance, the water table depth exceeds the level of $-0.25 \mathrm{~m}$ for 10 consecutive days or more on average only once every 10 years. Graphs like those of Figure 9 that are based on simulations of 30 year length are very helpful for design purposes and water management. Obviously, similar graphs can be calculated for average soil saturation and local drainage flux.

In this paper, only examples are given for three variables: water table depth, average soil saturation, and local drainage flux. However, the model estimates the complete water balance for the soil profile so that, if required, also sample functions of actual evapotranspiration can be simulated through (2), as well as groundwater recharge (precipitation excess minus change of storage in the unsaturated zone). However, the simulated actual evapotranspiration must be treated with care. This is because when assuming an equilibrium soil moisture profile, the model may overestimate the actual evapotranspiration in dry periods [Salvucci and Entekhabi, 1994].

\section{Summary and Conclusions}

In this paper a simple stochastic model was developed that is able to describe the nonlinear dynamics of the soil-groundwater system, without the need for solving Richards' equation. Its main goal is to describe accurately the fluctuations of shallow water tables. The model development resulted in a Stratonov- 
(a)

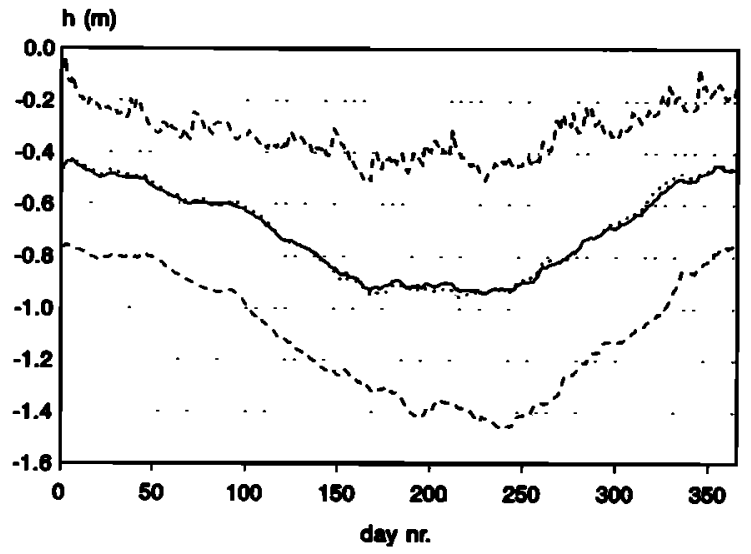

(b)

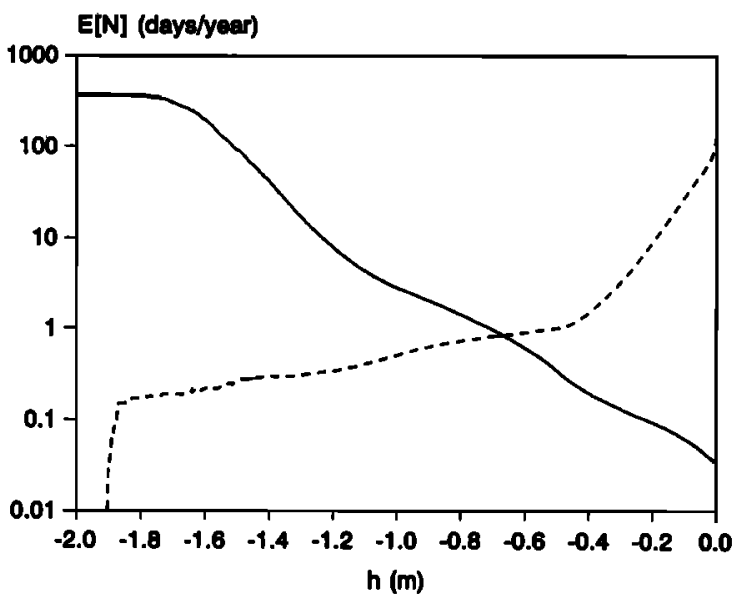

(c)

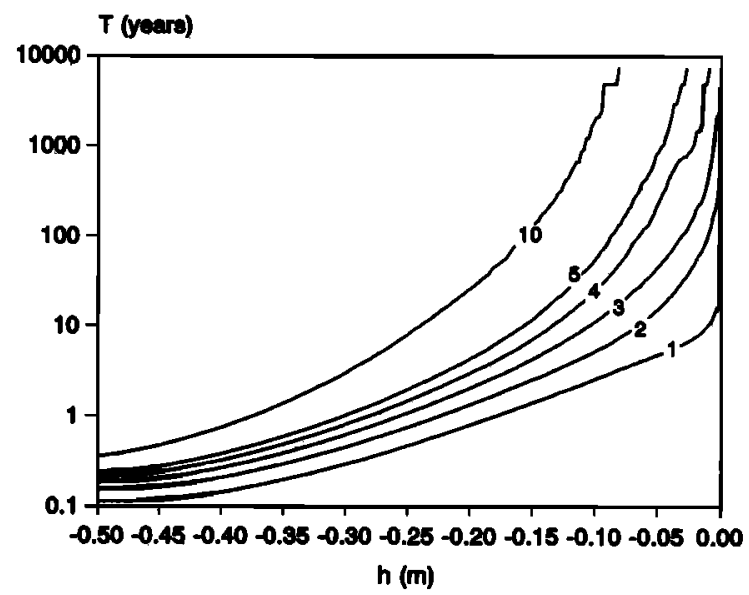

Figure 9. Measures of water table regimes estimated from 500 simulated sample functions of water table depth at location 12bl0015 for the period 1962-1991: (a) for every day number: mean (solid line), median (dotted line), 2.5 and 97.5 percentiles (dashed lines); (b) expected number of consecutive days that the water table depth is higher than a given level (solid) line, and lower than a given level (dashed line); (c) graphs showing the recurrence time of water table depth exceeding a given level for more than $1,2,3,4,5$, and 10 consecutive days.

ich-type stochastic differential equation (SDE) of water table depth at a single location. Inputs to the SDE are precipitation, potential evapotranspiration, a regional groundwater flux, and open water levels of nearby surface waters. The SDE can be used to generate alternative sample functions of water table depths and, derived from these, average soil saturation and local fluxes to/from nearby surface waters. A stationary solution of the probability density function (pdf) of water table depths was derived by means of the Fokker-Planck equation. This was achieved by modeling all inputs as constants with an additive white noise process. Although this is not a realistic representation of daily precipitation and potential evapotranspiration, the stationary solution can be used for very shallow water tables.

Some parameters of the SDE must be calibrated using time series of observed water table depths. For this purpose, the SDE was embedded in an extended Kalman filter, whose innovations are used to obtain maximum likelihood estimates of the calibrated parameters. The SDE was calibrated to (subsets of) time series of water table depths observed semi-monthly at two locations for a period of 10 years (1982-1991). At one location a peat soil is present with a very shallow water table, while at the other location a loamy sand soil is found with a moderately shallow water table. Validation showed that predictions made with the deterministic part of the model proved to be very close to the observations. Also, the magnitude of the prediction errors could be accurately estimated with the extended Kalman filter and the calibrated SDE.

Univariate statistics estimated from 1000 sample functions of daily water table depths, which were generated with the SDE, proved to be very close to those estimated from the measurements. The stationary pdf calculated with the FokkerPlanck equation underestimated the variation of the water table depths for the loamy sand soil. However, for the peat soil the stationary pdf was very close to that estimated from the measurements. This could be explained by the very small characteristic response time of the water table at the location of the peat soil. These results suggest that the stationary solution may be applicable to areas with very shallow water table tables and intensive drainage systems. 
Once the SDE is calibrated, it can be used to simulate sample functions of daily water table depth, average soil saturation, and local drainage flux for a time span that is much longer than the period for which measurements of the water table depth are available. In this way, drainage systems can be designed and operated based on time series that reflect the prevailing climate, instead of the weather conditions during the observation period. Moreover, multiple sample functions of the same period can be used for risk analyses. It is anticipated that the combination of the SDE with descriptions of soil profiles and surface elevation data will provide a tool for accurate space-time modeling of water table fluctuations.

\section{Appendix}

The derivative $(\partial b / \partial h)$ in (19) is given by

$$
\frac{\partial b}{\partial h}=\frac{-\sigma}{G^{2}(h)} \frac{\partial G}{\partial h}
$$

where $G(h)$ is given by (12) and ( $\partial G / \partial h)$ by the following expression:

$$
\frac{\partial G}{\partial h}=\frac{-\left(\theta_{s}-\theta_{r}\right)(n+1)\left(\alpha\left(z_{s}-h\right)\right)^{n}}{\left(z_{s}-h\right)\left[1+\left(\alpha\left(z_{s}-h\right)\right)\right]^{(2 n+1) / n}}
$$

The derivative ( $\partial a / \partial h)$ at time $t$ in (30) is given by

$$
\frac{\partial a}{\partial h}=\frac{G(h) \frac{\partial F}{\partial h}-F(h, t) \frac{\partial G}{\partial h}}{G^{2}(h)}
$$

with $G(h)$ given in (12) and ( $\partial G / \partial h)$ in (A2). $F(h, t)$ is given by

$$
F(h, t)=P(t)-F_{c} E_{p}(t)[S(h)]^{c}+q_{v}(t)-\sum_{i=1}^{m_{d}} \frac{h-H_{i}(t)}{\gamma_{i}}
$$

and its derivative by

$$
\frac{\partial F}{\partial h}=-c F_{c} E_{p}(t)[S(h)]^{c-1} \frac{\partial S}{\partial h}-\sum_{i=1}^{m_{d}} \frac{1}{\gamma_{t}}
$$

where in the summations only surface waters are included that are active for the given $h$. The average soil saturation $S(h)$ is given in (8) and its derivative $(\partial S / \partial h)$ by

$$
\frac{\partial S}{\partial h}=\frac{S(h)}{\left(z_{s}-h\right)}-\frac{S(h)}{\alpha^{n}\left(z_{s}-h\right)^{(n+1)}+\left(z_{s}-h\right)}
$$

Acknowledgments. C. P. Kim (Now at Boston Consulting Group BV, Baarn, the Netherlands) is thanked for fruitful discussions and ideas at the beginning of this Project. M. J. W. Jansen (DLO Agricultural Mathematics Group, Wageningen, the Netherlands) helped with some of the derivations. The help of A. W. Heemink (Faculty of Technical Mathematics and Informatics, Delft University of Technology, Delft, the Netherlands) with the development and application of the calibration procedure is gratefully acknowledged. C. B. M. te Stroet (Faculty of Civil Engineering, Delft University of Technology, Delft, Netherlands) and several colleagues at the DLO Winand Staring Centre, Wageningen, the Netherlands, are thanked for their comments on an earlier version of this manuscript. The comments of P. A. Troch (University of Ghent, Belgium), K. Hatfield (University of Florida, Gainesville), and an anonymous reviewer considerably improved the contents of this paper. This research was funded by DWK research programs 228 and 328 of the Netherlands Ministry of Agriculture, Nature Management and Fisheries.

\section{References}

Belmans, C., J. G. Wesseling, and R. A. Feddes, Simulation of the water balance of a cropped soil: SWATRE, J. Hydrol., 63, 271-286, 1983.

Box, G. E. P., and G. M. Jenkins, Time Series Analysis; Forecasting and Control, revised edition, Holden Day, San Francisco, Calif., 1976.

Deutsch, C. V., and A. G. Journel, GLSIB: Geostatistical Software Library and User's Guide, Oxford Univ. Press, New York, 1992.

Entekhabi, D., I. Rodriguez-Iturbe, and R. L. Bras, variability in largescale water balance with land surface-atmosphere interaction, J. Clim., 5(8), 798-813, 1992.

Feddes, R. A., Crop factors in relation to Makkink reference-crop evapotranspiration, in Commissie Hydrologisch Onderzoek TNO, Versl. Meded., vol. 39, pp. 33-45, CHO-TNO, The Hague, 1987.

Feddes, R. A., P. Kabat, P. J. T. Van Bakel, J. J. B. Bronswijk, and J. Halbertsma, Modelling soil water dynamics in the unsaturated zone-State of the art, J. Hydrol., 100, 69-111, 1988.

Gardiner, C. W., Handbook of Stochastic Methods for Physics, Chemistry and Natural Sciences, 2nd ed., Springer Verlag, New York, 1985.

Gehrels, J. C., F. C. van Geer, and J. J. de Vries, Decomposition of groundwater level fluctuations using transfer modelling in an area with shallow to deep unsaturated zones, J. Hydrol., 157, 105-138, 1994.

Hipel, K. W., and A. I. McLeod, Time Series Modelling of Water Resources and Environmental Systems, vol. 45, Dev. Water Sci., Elsevier Science, New York, 1994.

Jazwinski, A. H., Stochastic Processes and Filtering Theory, Academic, San Diego, Calif., 1970.

Knotters, M., and P. E. V. Van Walsum, Estimating fluctuation quantities from time series of water-table depths using models with a stochastic component, J. Hydrol., 197, 25-46, 1997.

Leduc, R, T. E. Unny, and E. A. McBean, Stochastic model of firstorder BOD kinetics, Water Res., 20(5), 625-632, 1986.

Lowry, W. P., The falling rate phase of evaporative soil moisture loss: A critical evaluation, Bull. Am. Meteorol. Soc., 40, 605, 1959.

Neuman, S. P., Feddes, R. A., and E. Bresler, Finite element simulation of flow in saturated-unsaturated soils considering water uptake by plants: Development of methods, tools and solutions for unsaturated flow, 3rd annual report, part 1, Israel Inst. of Technol., Haifa, 1974.

Newton, N. J., Asymptotically efficient Runge-Kutta methods for a class of Itô and Stratonovich equations, SLAM J. Appl. Math., 51(2), 542-567, 1991.

Papoulis, A., Probability, Random Variables and Stochastic Processes, 3rd ed., McGraw-Hill, New York, 1991.

Press, W. H., B. P. Flannery, S. A. Teukolsky, and W. T. Vetterling, Numerical Recipes: The Art of Scientific Computing, Cambridge Univ. Press, New York, 1986.

Sage, A. P., and J. L. Melsa, Estimation Theory With Applications to Forecasting and Control, McGraw-Hill, New York, 1976.

Salvucci, G. D., and D. Entekhabi, Equivalent steady soil moisture profile and the time compression approximation in water balance modeling, Water Resour. Res., 30(10), 2737-2749, 1994.

Schweppe, F. C., Uncertain Dynamic Systems, Prentice-Hall, Englewood Cliffs, N. J., 1973.

Stratonovich, R. L., Topics in the Theory of Random Noise, vol. 2, translated from Russian by R. A. Solverman, Gordon Breach, New York, 1967.

Tankersley, C. D., W. D. Graham, and K. Hatfield, Comparison of univariate and transfer function models of groundwater fluctuations, Water Resour. Res., 29(10), 2517-3533, 1993.

te Stroet, C. B. M., Calibration of stochastic groundwater flow models: Estimation of system noise statistics and model parameters, Ph.D. thesis, Delft Univ. of Technol., Delft, Netherlands, 1995.

Troch, P. A., Conceptual basin-scale runoff process models for humid catchments: Analysis, synthesis and applications, Ph.D. thesis, Ghent Univ., Ghent Belgium, 1992.

Troch, P. A., F. P. De Troch, and W. Brutsaert, Effective water table depth to describe initial conditions prior to storm rainfall in humid regions, Water Resour. Res., 29(2), 427-434, 1993.

Unny, T. E., Numerical integration of stochastic differential equations in catchment modeling, Water Resour. Res., 20(3), 360-368, 1984. 
Van der Sluijs, P., and J. J. De Gruijter, Water table classes: A method to describe seasonal fluctuation and duration of water tables on Dutch soil maps, Agric. Water Manage., 10, 109-125, 1985.

van Geer, F. C., and A. F. Zuur, An extension of Box-Jenkins transfer/ noise models for spatial interpolation of groundwater head series, $J$. Hydrol., 192, 65-80, 1997.

Van Genuchten, M. T., A closed-form equation for predicting the hydraulic conductivity of unsaturated soils, Soil Sci. Soc. Am. J., 44, 892-898, 1980

Winter, T. C., D. O. Rosenberry, and A. M. Sturrock, Evaluation of 11 equations for determining evaporation for a small lake in the north central United States, Water Resour. Res., 31(4), 983-993, 1995.
Wong, E., and M. Zakai, On the relation between ordinary and stochastic differential equations, Int. J. Eng. Sci., 3, 213-222, 1965.

Wösten, J. H. M., and M. T. Van Genuchten, Using texture and other soil properties to predict the unsaturated hydraulic conductivity, Soil Sci. Soc. Am. J., 52(6), 1762-1770, 1988.

M. F. P. Bierkens, DLO, Winand Staring Centre for Integrated Land, Soil and Water Research, P.O. Box 125, 6700 AC Wageningen, Netherlands. (bierkens@sc.dlo.nl)

(Received May 4, 1998; revised June 30, 1998; accepted July 9,1998 .) 